arrhythmic properties were recognised. Its effects include bradycardia, augmentation of coronary blood flow, and prolongation of the action potential of atrial and ventricular muscle. In 1973 Coumel and Bouvrain" reported that amiodarone was highly effective in treating various arrhythmias including those complicating the WPW syndrome. Rosenbaum et $\mathrm{al}^{3}$ later achieved total suppression of arrhythmias in al of 27 cases with this condition. A wide variety of other atrial and ventricular arrhythmias can also be controlled, even in patients with severe congestive failure. ${ }^{3}$

The beneficial effect of amiodarone in atrial fibrillation and on conduction in anomalous pathways is related to the prolongation of action potential and refractory period of the myocardium. The drug is cumulative with a slow rate of elimination. ${ }^{4}$ The onset of action is slow and persists for weeks after dosage has ended. ${ }^{3}$ These properties explain the continued success of amiodarone in our case when dosage was reduced to $200 \mathrm{mg}$ on alternate days. Microdeposits occur in the corneae $^{5}$ but, in the dosage used to control arrhythmias, rarely cause symptoms $s^{3}$ and are reversible. The drug has not yet been licensed in Britain by the Medicines Commission.

In this case amiodarone produced a dramatic and life-saving effect, restoring a severely incapacitated man to excellent health after the reversion of previously refractory atrial fibrillation to sinus rhythm.

Dr Dennis Krikler suggested to us in 1973 that the electrophysiological properties of amiodarone seemed appropriate for the management of refractory arrhythmias in WPW syndrome. Amiodarone (Labaz, Brussels) was supplied by Dr R Smith of Reckitt and Colman.

${ }^{1}$ Wellens, H J, and Durrer, D, Circulation, 1973, 47, 1229.

2 Coumel, P, and Bouvrain, Y, Le fournal des Agrégés, 1973, 6, 69

${ }^{3}$ Rosenbaum, M B, ei al, American fournal of Cardiology, 1976, 38, 934.

${ }^{4}$ Broekhuysen, J, Laruel, R, and Sion, R, Archives internationales de pharmacodynamie et de thérapie, 1969, 177, 340 .

${ }^{5}$ Verin, $\mathrm{Ph}$, et al, Archives d'ophtalmologie et revue générale d'ophtalmologie, $1971,31,581$.

(Accepted 25 August 1977)

Royal Sussex County Hospital, Brighton BN2 5BE

D A CHAMBERLAIN, MD, FRCP, consultant cardiologist

Brighton General Hospital, Brighton BN2 3EW

A N G CLARK, MD, FRCP, consultant geriatrician

\section{Diurnal variation of serum urate in pregnancy}

The degree of hyperuricaemia is widely used as a prognostic index in pre-eclampsia. ${ }^{1}$ Nevertheless, the reliability of the random blood sample conventionally used for this has not been adequately assessed during pregnancy, despite evidence in non-pregnant women of considerable variation from day to day and within any given day. ${ }^{2-4}$ We therefore decided to examine changes in serum urate concentration in normal pregnant women throughout a 24 -hour period.

\section{Patients, methods, and results}

Fourteen normotensive inpatients were studied without restriction of diet or activity throughout 24 hours during the third trimester of pregnancy. One patient (case 3 ) was carrying twins. No patient was taking medication known to affect the serum urate concentration. Venous blood samples were withdrawn at $0600,1000,1400,1800$, and 2200 on day 1 and at 0600 the next morning. Serum was analysed on the day of collection, or snap-frozen in liquid nitrogen and stored at $-20 \mathrm{C}$ for not more than three weeks. This procedure had been validated," and its reliability was confirmed in our laboratory. Duplicate samples were analysed manually by an enzymatic method based on a commercial reagent pack (Uricaquant). The mean error of the method was $\pm 1.5^{\circ}$.

The interpatient variability was much greater than the intersample variability in any patient (see table). The overall mean and the standard deviation within samples within patients showed that the variability among random samples was small, but a range of up to $30^{\circ}$, was found in some women. No consistent pattern of variation emerged, but there were significant differences between certain of the time points. A longer study, however, would be needed to validate these differences.

\section{Comment}

The results suggest that pregnant women have considerable fluctuations of serum urate during the day. Despite a tendency towards lower concentrations during daylight hours, no single reading would be representative for any individual. These results agree with published data in non-pregnant women.

Furthermore, serum urate concentration does not appear to remain stable from day to day; comparison of samples taken 24 hours apart again shows considerable variation within individuals. In the data presented the second of the results is lower than the first in most cases. This unexpected pattern has not been confirmed in subsequent studies.

Intersample variability is itself of different magnitude from patient to patient, so that it would not be reasonable to predict the accuracy of a given result on the basis of the concentration of urate reported. Thus a single blood sample in the individual case may not truly represent the mean value for that patient throughout the day. This is particularly relevant to the popular practice of screening pregnant women for hyperuricaemia at a routine antenatal visit.

The cause of this diurnal variation remains unexplained. The overall pattern does not suggest a relation between urate and purine ingestion at meals. One factor may be an alteration in urate excretion, and this is being investigated.

${ }^{1}$ Redman, C W G, et al, Lancet, 1976, 1, 1370.

2 Brachner-Mortensen, K, Acta Medica Scandinavica, 1937, Supp, 84, 13.

3 Zachau-Christiansen, B, Scandinavian fournal of Clinical and Laboratory Investigation, 1957, 9, 244

${ }^{4}$ Rubin, R T, et al, fournal of the American Medical Association, 1969, 208, 1184 .

${ }^{5}$ Liddle, L, Seegmiller, J E, and Lester, L, fournal of Laboratory and Clinical Medicine, 1959, 54, 903.

(Accepted 6 September 1977)

MRC Reproduction and Growth Unit and Department of Obstetrics and Gynaecology, Princess Mary Maternity Hospital, Newcastle upon Tyne NE2 3BD

LYNDON M HILL, MD, visiting research fellow, MRC Unit

CHRISTINE FURNESS, technician

WILLIAM DUNLOP, MRCOG, FRCSED, senior lecturer in obstetrics

\begin{tabular}{|c|c|c|c|c|c|c|c|}
\hline Case No & 0600 & 1000 & 1400 & 1800 & 2200 & 0600 & Mean $\pm S D$ \\
\hline $\begin{array}{r}1 \\
2 \\
3 \\
4 \\
5 \\
6 \\
7 \\
8 \\
9 \\
10 \\
11 \\
12 \\
13 \\
14\end{array}$ & $\begin{array}{l}178 \\
184 \\
351 \\
304 \\
193 \\
343 \\
184 \\
235 \\
193 \\
242 \\
330 \\
287 \\
231 \\
233\end{array}$ & $\begin{array}{l}170 \\
180 \\
344 \\
310 \\
207 \\
336 \\
169 \\
244 \\
184 \\
261 \\
323 \\
281 \\
233 \\
235\end{array}$ & $\begin{array}{l}163 \\
174 \\
353 \\
298 \\
199 \\
347 \\
144 \\
210 \\
168 \\
253 \\
291 \\
271 \\
219 \\
210\end{array}$ & $\begin{array}{l}158 \\
158 \\
316 \\
281 \\
187 \\
327 \\
137 \\
208 \\
174 \\
240 \\
277 \\
268 \\
198 \\
191\end{array}$ & $\begin{array}{l}162 \\
155 \\
320 \\
265 \\
175 \\
320 \\
128 \\
211 \\
187 \\
227 \\
269 \\
254 \\
191 \\
188\end{array}$ & $\begin{array}{l}170 \\
162 \\
318 \\
265 \\
196 \\
308 \\
132 \\
224 \\
186 \\
254 \\
288 \\
273 \\
203 \\
215\end{array}$ & $\begin{array}{l:l}166 \cdot 8 & 7 \cdot 2 \\
168 \cdot 8 & 12 \cdot 1 \\
333 \cdot 7 & 17 \cdot 5 \\
287 \cdot 2 & 19 \cdot 7 \\
192 \cdot 8 & 11 \cdot 0 \\
330 \cdot 2 & 14 \cdot 7 \\
149 \cdot 0 & 22 \cdot 5 \\
222 \cdot 0 & 15 \cdot 0 \\
182 \cdot 0 & 9 \cdot 2 \\
246 \cdot 2 & 12 \cdot 3 \\
296 \cdot 3 & 24 \cdot 8 \\
272 \cdot 3 & 11 \cdot 4 \\
212 \cdot 5 & 17 \cdot 7 \\
212 \cdot 0 & 20 \cdot 0\end{array}$ \\
\hline Mean $\pm S D$ & $249 \cdot 1+62 \cdot 5$ & $248 \cdot 4: 62.5$ & $235 \cdot 7: 67 \cdot 7$ & $222 \cdot 9: 61 \cdot 7$ & $218 \cdot 0: 59 \cdot 9$ & $228 \cdot 1 \div 57 \cdot 3$ & $233 \cdot 7+16 \cdot 1$ \\
\hline
\end{tabular}

